\title{
A GRAIN BOUNDARY PHASE TRANSITION STUDIED BY MOLECULAR DYNAMICS
}

\author{
P. DEYMIER, $\dagger$ A. TAIWO and G. KALONJI \\ Department of Materials Science and Engineering, Massachusetts Institute of Technology, \\ Cambridge, MA 02139, U.S.A.
}

(Received 30 May 1985; in revised form 2 February 1987)

\begin{abstract}
We have conducted a thorough investigation of the high temperature behaviour of crystalline interfaces in 2-dimensional close-packed and 3-dimensional f.c.c. $\Sigma=7$ bicrystals using the atomistic computer simulation technique of molecular dynamics. Gibbs free energies of the bicrystals were computed using the determinant technique and a computer calorimetry technique which we describe. The thermodynamic properties of the boundary were monitored over a wide range of temperatures, up to the bulk melting point. We observed a first order phase transition of the grain boundary in the $2 \mathrm{D}$ and $3 \mathrm{D}$ bicrystals at temperatures well below the bulk melting temperature. At the transition temperature the crystalline grain boundaries are replaced by highly disordered liquid-like layers.
\end{abstract}

\begin{abstract}
Résumé-Nous avons etudie par la methode de simulation en dynamique moleculaire les propriétés a haute temperature d'interfaces cristallines $\Sigma=7$ a grand angle de desorientation dans des bicristaux bidimensionnels dans le plan compacte et dans des bicristaux c.f.c. tridimensionnels. Nous decrivons les techniques du "determinant" et de "distribution des energies" que nous avons employées pour calculer l'enthalpie libre des bicristaux. Les propriétés thermodynamiques des interfaces sont calculees dans un large intervalle de temperature jusqu'à la temperature de fusion du cristal parfait. Les interfaces cristallines dans les bicristaux bidimensionnels et tridemensionnels subissent une transformation de phase du premier ordre a une température inférieure a la température de fusion du cristal parfait. A la température de transition, l'interface cristalline se transforme en une region hautement desordonnée similaire à un liquide.
\end{abstract}

\begin{abstract}
Zusammenfassung-Das Hochtemperaturverhalten kristalliner Grenzflächen in 2-dimensionalen dichtestgepackten und 3-dimensionalen $\mathrm{kfz} . \Sigma=7$-Bikristallen wurde mittels der atomistischen Simulationstechnik der molekularen Dynamik sorgfältig untersucht. Die freie Enthalpie der Bikristalle wurde mit einem determinanten- und einem kalorimetrischen Computerverfahren, die beschrieben werden, berechnet. Die thermodynamischen Eigenschaften der Korngrenze werden in einem weiten Temperaturbereich bis zum Schmelzpunkt des Festkörpers ermittelt. Im 2- und im 3-dimensionalen Kristall wurde ein Phasenübergang erster Ordnung weit unterhalb dieser Schmelztemperatur beobachtet. Bei der übergangstemperatur wird die kristalline Korngrenze durch eine stark ungeordnete flüssigkeitsähnliche Schicht ersetzt
\end{abstract}

\section{INTRODUCTION}

The role of crystalline defects in mediating transitions in and among bulk crystalline phases is well known. There exists also, however, the logical possibility, which is refiected increasingly in nature as a physical rcality, that defects may undergo phase transitions of their own under circumstances in which their bulk crystalline hosts are stable. The possibilities for phase transitions of defects are rich and diverse, largely because of the functional dependence of the thermodynamic properties of the defects on certain geometrical parameters, in addition to those more commonly encountered in the thermodynamics of bulk phases. These parameters describe the operations involved in the idealized construction of the defects from the crystalline matrices in which they reside.

†Present address: Department of Materials Science and Engineering, University of Arizona, Tuscon, AZ 85721, U.S.A.
Our research interests lie in the area of phase equilibria of crystalline interfaces. The external surfaces of crystals are more easily accessible experimentally than internal interfaces, such as grain boundaries, antiphase boundaries and crystal-crystal interphase boundaries. In the surface science community a rich and rapidly growing literature attests to the diversity of phase transitions that external surfaces may undergo. In the field of internal interfaces, however, systematic knowledge of their phase equilibria is much more limited. Because of the overwhelming importance of interfacial phenomena for the understanding of the physical properties of the bulk defected solid state, there is a great need for systematic studies of the thermodynamics of interfaces. We have chosen to undertake such a study using the atomistic computer simulation technique of molecular dynamics.

In this paper we report the results of a thermodynamic investigation of a high-angle coincidence tilt boundary $(\Sigma=7)$. We have studied both 2 - and 3-dimensional $\Sigma=7$ boundaries, in which the atoms 
were interacting through a Lennard-Jones potential. Our simulations were performed using an isostressisothermal formulation of molecular dynamics which allows the simulation cell to change both size and shape, and which is, therefore, particularly wellsuited for the study of phase transitions. To address the question of grain boundary phase equilibria at finite temperatures we need knowledge of grain boundary excess free energies. For this reason, we worked on developing and implementing techniques for direct evaluation of grain boundary free energies using molecular dynamics. We have monitored the behaviour of the thermodynamic properties of our boundaries through a wide range of temperatures; from low temperatures to the bulk melting point.

One of the striking results of our study is the occurence of a phase transition in our grain boundaries which takes place at temperatures below $80 \%$ of the bulk melting temperature. In this transition a periodic, crystalline grain boundary is replaced by a highly disordered liquid-like layer. We have characterized the transition through the behaviour of the calculated excess enthalpy, volume and Gibbs free energy of the boundary. The calculated transition is first order for boundaries in both the 2-dimensional and the 3-dimensional bicrystals.

The organization of the paper is as follows. We discuss briefly the current knowledge concerning phase transitions of grain boundaries, both experimental and theoretical, in Section 2. A description of the computational techniques employed follows in Section 3 , including discussion of the flexible borders formulation of molecular dynamics, and the techniques employed for calculation of free energies. The results of our simulations, for both the two dimensional and the three dimensional systems, are presented in Section 4. Discussion and conclusions are contained in Section 5.

\section{GRAIN BOUNDARY PHASE EQUILIBRIA}

The conceptual description of a grain boundary as a distinctive entity which may undergo specific structural mutations independently of the bulk phase is a consequence of Gibbs's classical development of surface thermodynamics [1]. An extensive thermodynamic property of an interface is simply defined as an excess interfacial quantity. The excess value is given by the difference between the value of the property in a system containing the defect and its value in a reference perfect crystal system under the same equilibrium conditions. A grain boundary phase transition is defined in terms of discontinuities in the first derivatives of the excess Gibbs free energy for first-order transitions and discontinuities in the higher-order derivatives of the excess Gibbs free energy for higher-order grain boundary phase transitions.

All of the properties of a grain boundary depend on a number of geometrical parameters, involved in the idealized construction of the boundary. In addition to the more typical thermodynamic variables, such as temperature, pressure and chemical potential, we need ten geometrical variables to completely specify a planar grain boundary. Three angular variables specify the rigid body misorientation $\mathbf{R}$ between the grains. Three variables describe any relative rigid body translation $t$ between the grains. Three more variables specify the exact locations of the interface plane with respect to both crystals. Finally, one variable describes the chirality of the rotational operation $\mathbf{R}$, in order to permit one to distinguish enantiomorphs of the same phase.

The crystalline interface in its search for minimum frec cnergy is free to relax any of the geometrical parameters, except for the one describing the chirality. A grain boundary phase equilibrium diagram is then represented in a $12+C$ dimensional space, where $C$ is the number of independent chemical components. Phase transformations of grain boundaries will, in general, involve changes in the values of the geometrical parameters. An interesting example is the facetting transition of grain boundaries, a transition involving variation of the parameters describing the boundary normal, and which may take place at fixed values of the misorientation and relative translation states, $\mathbf{R}$ and $\mathbf{t}$. Interesting theoretical and experimental work on facetting transitions of crystal-vapour surfaces, and of grain boundaries has been accomplished [2,3].

There exists also, under special circumstances often dictated by symmetry, the possibility of grain boundary phase transitions that do not involve changes in the values of the geometrical parameters. These transitions may be called congruent grain boundary phase transitions. A particularly interesting transition, for which there exists some theoretical and experimental evidence, is the grain boundary melting transition. In this transition a well-ordered, periodic boundary is replaced by a highly disordered liquidlike layer at temperatures significantly below the bulk melting point. A subset of grain boundary melting transitions, called congruent melting transitions of grain boundaries, can take place without changes in the values of the geometrical parameters. A recent study has examined the conditions under which grain boundary melting can take place without facetting of the grain boundary [4]. A direct analogy can be drawn between the role of the compositional variables in melting of crystalline phases and the role of the variables specifying the normal in the melting of grain boundaries. At a congruent grain boundary melting point the free energy surfaces of the grain boundary phases touch without intersecting. Prime candidates for congruent melting are therefore grain boundaries for which the Gibbs free energy is a local minimum with respect to variation of the geometrical parameters.

Symmetry arguments can be very useful in pinpointing values of the geometrical parameters for 
which the free energy, and all the other properties of the grain boundary, must be at a local extremum. Boundaries having these values of the parameters are said to be at symmetry-dictated extrema with respect to variation of the geometrical parameters [5]. Such boundaries are then logical ones to choose for the study, buth experimental and theoretical, of congruent phase transitions of grain boundaries.

It is well established that external surfaces undergo phase transitions [6, 7]. However, the experimental knowledge of grain boundary phase transitions is in an inconclusive state. Willens et al. have reported experimental evidence for a $\mathrm{Pb}$ melting point suppression and broadening in modulated $\mathrm{Pb} / \mathrm{Ge}$ foils [8]. The origin for the onset of melting well below the bulk melting temperature of $\mathrm{Pb}$ and of the broadening was not understood, but could have resulted from a grain boundary melting transition. Glicksman and Vold have found a congruent grain boundary melting transformation in bismuth using beam heating in a transmission electron microscope [9]. Early work on grain boundary sliding for high angle tilt boundaries in pure $\mathrm{Al}$ [10], and more recently in pure $\mathrm{Zn}[11]$, provided indications of the existence of some sort of structural transformation taking place in the boundaries at temperatures well below the bulk melting point. The velocity of grain boundary sliding was measured in well-characterized bicrystals, of various misorientations, as a function of temperature. A discontinuity in the rate of sliding was observed at a transition temperature, which depends to a large extent on the misorientation of the grains, and which ranges from approximately 70 to $90 \%$ of the bulk melting point. The temperature at which the discontinuity in sliding rate was observed was higher for coincidence grain boundaries than for general boundaries. This may be accounted for by considering the fact that the melting temperature of a highly symmetrical congruent phase is greater than the melting temperature of a boundary off the congruent structure. However, these studies provided no structural charactcrization of the transformed boundary, lcaving some unresolved questions as to the nature of the transition.

On the computational side, Kikuchi and Cahn observed a gradual melting of a symmetric $\Sigma=5$ boundary in a 2-dimensional square lattice-gas model [12]. The boundary was gradually replaced by a low density liquid-like layer far below the bulk melting point. The gradual character of the melting was attributed to the dimensionality of the system. Another gradual grain boundary disordering was discovered in a 3-dimensional Lennard-Jones f.c.c. bicrystal containing two $\Sigma=5$ tilt boundaries [13]. The transition was characterized through calculation of local short and long-range order parameters in the boundary region and through the behaviour of the grain boundary diffusion coefficient. The interpretation of the gradual character of the transition was based on the continuous variation to the liquid value, as the temperature was raised, of these calculated quantities. These simulations were performed at constant volume causing the pressure of the system to reach very high values, and making a comparison with other simulation results difficult. As pointed out by the authors [13] the behavior of the boundary in the temperature region studied is compatible with a description of the grain boundary as a crystalline solid with a high point defect concentration. The highest temperature investigated was only $0.76 T_{\mathrm{m}}$, and we believe that the reported behaviour was not a boundary melting transition. We expect that a grain boundary melting transition would occur in this system at a temperature closer to the bulk melting temperature $T_{\mathrm{m}}$.

In previous preliminary papers we have discussed the onset of grain boundary melting in two dimensional $\Sigma=7$ and $\Sigma=13$ bicrystals $[14,15]$. We studied these transitions through the temperature dependence of bicrystal volume and enthalpy calculated from molecular dynamics simulations in the $(N, P, T)$ ensemble. We observed discontinuities in these calculated bicrystal properties at temperatures well below the calculated bulk melting temperature. Structural observations of the systems revealed the appearance of a highly disordered liquid-like layer at the transition temperature. A similar transition has recently been observed in molecular dynamics simulations of a $\Sigma=5$ grain boundary in which the particles were interacting through a Morse potential for aluminum [16]. In another study, interface melting in various tilt boundaries of Lennard-Jones particles was predicted by a harmonic analysis of the ground state configurations [17]. The high temperature structures obtained by molecular dynamics by these authors also exhibit what appear to be melted layers at the boundaries. There remains, however, disagreement as to the nature of the phase transition occurring in these systems. In the present study we address this question through explicit calculation of the temperature dependence of the grain boundary excess thermodynamic properties, and in particular, the excess grain boundary free energy. We conclude that the grain boundary phase transition we observe is a bona fide first order interfacial phase transition.

\section{COMPUTATIONAL METHODS}

In this section we describe the details of the computational methods involved in our simulations. We begin by discussing the isostress-isothermal formulation of molecular dynamics. This is followed by a discussion of methods for calculating free energies of grain boundaries.

\subsection{Molecular dynamics in the $(N, \sigma, T)$ ensemble}

The constraint of constant volume in standard molecular dynamics (MD) in the canonical ensemble has been removed recently allowing the possibility of simulating periodic cells of fluctuating volume and 
shape. This formulation is extremely useful for the study of grain boundary phase transitions, because of the changes in volume and shape that, in the general case, accompany these transitions. Molecular dynamics in the isothermal-isostress ensemble is based on an extension by Parrinello and Rahman [18] of a Lagrangian formulation due to Andersen [19] for a system with flexible borders.

Three variable vectors, a, b, c forming a $3 \times 3$ matrix $h$ are necessary to describe the planar borders of the simulation cell whose variable volume is therefore $\Omega=\mathbf{a} \cdot(\mathbf{b} \times \mathbf{c})$. A position vector $\mathbf{r}_{i}$ of a particle $i$ in a cartesian coordinate system may be expressed in the $(a, b, c)$ coordinate system as $\mathbf{s}_{i}=\mathbf{h}^{-1} \mathbf{r}_{i}$.

The Hamiltonian of this system under constant stress is

$$
H=\frac{1}{2} m \sum_{i} \mathbf{v}_{i}^{2}+\sum_{i>j} U\left(\mathbf{r}_{i j}\right)+\frac{1}{2} W \operatorname{Tr}^{\prime} \dot{\mathbf{h}}+\operatorname{Tr}\{\sigma \epsilon\} \Omega_{0} .
$$

In the above equation where $h$ stands for the time derivative of the matrix $h$, and $h^{-1}, h^{t}$, and Trh are, respectively, the inverse, transpose and trace of the matrix $h$. The total energy is the sum of the kinetic and potential energies of the set of atoms, the kinetic energy of the borders, to which one artificially assigns a mass $W$, and the elastic energy, for which $\Omega_{\mathrm{o}}$ is the volume of the reference system used to measure the strain $\epsilon$. To stay in the limit of small displacements, the reference state is chosen as $\mathbf{h}_{\mathbf{0}}=\langle\mathbf{h}\rangle_{\Delta t}$, where the elements of the $h_{0}$ matrix are the coordinates of the $\mathbf{a}, \mathbf{b}, \mathbf{c}$ vectors averaged over a period of time $\Delta t$. This reference state for displacement is used over the subsequent interval of time $[\Delta t, 2 \Delta t]$ while a new reference state is computed. The displacement $\mathbf{u}$ due to the distortion $\mathbf{u}=\mathbf{r}-\mathbf{r}_{0}=\left(\mathbf{h h}_{0}^{-1}-1\right) \mathbf{r}_{0}$ leads to the strain tensor $\epsilon=1 / 2\left(h_{0}^{t-1} \mathbf{G h}_{0}^{-1}-1\right)$, with $\mathbf{G}=\mathbf{h}^{\prime} \mathbf{h}$. After dropping the constant terms which are inconsequential for the dynamical equations, the elastic energy is $E_{\mathrm{el}}=(1 / 2) \operatorname{Tr}\{\boldsymbol{\Sigma} \mathbf{G}\}$, where $\Sigma=\mathbf{h}_{0}^{-1} \sigma \mathbf{h}_{0}^{t-1} \Omega_{0}$, which is a symmetric tensor related to the applied stress $\sigma$.

The equations of motion derived from this Hamiltonian are

$$
\ddot{\mathbf{s}}_{i}=-1 / m \sum\left(1 / \mathbf{r}_{i j}\right)\left[\partial Y\left(\mathbf{r}_{i j}\right) \partial \mathbf{r}_{i j}\right]\left(\mathbf{s}_{i}-\mathbf{s}_{j}\right)-\mathbf{G}^{-1} \mathbf{G} \mathbf{G} \mathbf{s}_{i}
$$

with $i, j=1,2,3, \ldots N$, and

$$
W \grave{h}-(\pi-p \mathbf{I}) \Omega \mathbf{h}^{t-1}-\mathbf{h} \Sigma .
$$

In the above expression, $\pi$, the internal stress matrix, is defined by

$$
\pi=\frac{1}{\Omega}\left\{\sum_{i} m_{i} \mathbf{v}_{i} \mathbf{v}_{i}-\sum_{i} \sum_{j>i}\left(\frac{1}{r_{i j}}\right)\left(\frac{\partial U}{\partial r_{i j}}\right) \mathbf{r}_{i j} \mathbf{r}_{i j}\right\} .
$$

The time average of any function calculated from the trajectories solved from these equations of motion is equal to an ensemble average in an isenthalpicisostress ensemble. The equipartition of energy theorem states that the temperature will adjust itself to maintain a constant Hamiltonian. Adding energy to the system by rescaling the atomic velocities to fix the temperature results in the generation of trajectories on which the time average is an average in the $(N, \sigma, T)$ ensemble. Many thermodynamic properties (including pressure, volume, temperature, and internal energy) are directly obtainable as time averages over these trajectories, if the system remains in a particular macroscopic state for sufficiently long times. The calculation of entropy, and hence, free energies, however, cannot be performed in so simple a fashion, because entropy is a collective property and is not instantaneously defined. To calculate entropy one must have knowledge of the partition function of the system. Techniques for overcoming this difficulty are discussed below.

The equilibrium properties of a system are independent of the mass of its constituents. Thus the quantity $W$, which represents the mass of the borders of the simulation cell and which has no physical reality, is chosen arbitrarily on the basis of computational convenience. We have taken $W$ such that the relaxation time from an imbalance between external and internal stresses is small. The usual features of standard molecular dynamics such as periodic boundary conditions, truncation of the interatomic potential, and Maxwell-Boltzman distribution of initial velocities were used in our simulations.

\subsection{Techniques for the calculation of free energies}

3.2.1. The determinant technique. This technique, due to Hoover [2], consists of a direct evaluation of the partition function of the system within the quasi-harmonic approximation. The simple harmonic approximation relies upon the truncation to second order of the Taylor expansion of the potential energy. It is assumed that the effect of higher order terms leading to thermal expansion can be taken into account by using equilibrium atomic positions at $T>0 K$ for the series expansion. This is the essence of the quasi-harmonic approximation. Under this approximation, the total potential energy of the system can be written as a quadratic from in the particle displacements, $\mathbf{u}_{i}$

$$
U=U_{0}+(1 / 2) \Sigma k_{i j} \mathbf{u}_{i} \mathbf{u}_{j}
$$

where $k_{i j}$ is a force constant coefficient and $U_{0}$ the static lattice potential energy. The Helmoltz free energy $F$, is related to the canonical partition function $Q$ by

$$
F=-k T \ln Q
$$

with

$$
Q=\left(2 \pi m k T / h^{2}\right)^{D N / 2} \mathrm{e}^{-U(\mathbf{r}) / k T} \mathrm{~d} \mathbf{r} .
$$

$D$ is the dimensionality of the system. The partition function $Q$, which can be written as

$$
\begin{aligned}
Q=\left(2 \pi m k T / h^{2}\right)^{S N / 2} & \exp \left(-U_{0} / k T\right) \\
& \times \int \exp \left[(-1 / 2 k T) \sum k_{i j} \mathbf{u}_{i} \mathbf{u}_{j}\right] \mathrm{d} \mathbf{u}
\end{aligned}
$$


is then proportional to the inverse square root of the determinant of the matrix of the force constant coefficients $\left[k_{i j}\right]$

$$
\begin{aligned}
Q=\left(2 \pi m k T / h^{2}\right)^{D N / 2} & \exp \left(-U_{0} / k T\right) \\
& \times(2 \pi k T)^{D N / 2}\left\{\operatorname{Det}\left[k_{i j}\right]\right\}^{-1 / 2} .
\end{aligned}
$$

The determinant of this matrix vanishes due to the existence of displacements for which no restoring forces are involved, such as global rotation or translation of the system. Under periodic boundary conditions only the latter remains. This translational motion is then constrained by pinning one particle (setting $\mathbf{u}_{1}=0$ for particle 1 , say). the partition function under this restriction becomes

$$
\begin{aligned}
Q=\left(2 \pi m k T / h^{2}\right)^{D N / 2} & \exp \left(-U_{0} / k T\right) \Omega \\
& \times(2 \pi k T)^{D(N-1) / 2}\left\{\operatorname{Det}\left[k_{l}^{\prime}\right]\right\}^{-1 / 2} .
\end{aligned}
$$

The new determinant to evaluate is the determinant of a $D(N-1) \times D(N-1)$ matrix built from the original $D N \times D N$ force constant matrix by removing rows and columns corresponding to the fixed particle (i.e. wherever 1 appears as a subscript in $k_{i j}$ ). The force constant coefficients are calculated from an average structure generated in a $M D$ simulation with the expression

$$
k_{i j}=\frac{\partial^{2} U\left(r_{i j}\right)}{\partial r_{i} \partial r_{j}} \mathbf{r}_{i}^{0} \mathbf{r}_{j}^{0}
$$

evaluated at the equilibrium positions $\mathbf{r}_{i}^{0}, \mathbf{r}_{j}^{0}$, when $i \neq j$. The self force constants $k_{i i}$ are obtained by the sum rule [21]

$$
k_{i i}=-\sum_{j \neq i} k_{i j}
$$

The determinant technique has limitations intrinsic to the quasi-harmonic approximation. It gives only the vibrational contribution to the free energy and is effective only in temperature ranges where reliable average structures are accessible. On the other hand, at very low temperatures the energy levels are quantized and the classical form of the canonical partition function is not valid any more. If needed, quantum corrections can be taken into account [22].

3.2.2. Energy Distribution Methods. These methods were first introduced by Valleau et al. [23] and Bennett [24]. They were initially developed for the Monte Carlo algorithm but can be utilized as well with the MD technique. The heart of the energy distribution methods is that one is able to express ratios of partition functions between a system of interest and a reference system without knowing the functions themselves. The reference system may differ from the system of interest not only in its thermodynamic state variables (e.g. temperature) but also in its Hamiltonian (e.g. potential energy function). The former will give the free energy difference for the same system at two temperatures; the latter, the free energy difference of two systems at the same tem- perature. Because of their similarity to calorimetry, these methods are sometimes called computer calorimetry. In this paper, we discuss only the method for calculating the free energy difference of a system at two temperatures. We developed all the expressions in the isothermal-isobaric ensemble but they can be written in other ensembles without many modifications. In the (N, P, T) ensemble the thermodynamic potential is the Gibbs free energy which is related to $\Delta$, the isothermalisobaric partition function by

$$
G=-k T \ln \Delta .
$$

A two-sided evaluation of the free energy difference between two identical systems held at different temperatures is based on the construction of two normalized distribution functions $h_{0}(x)$ and $h_{1}(x)$, which are given by

$$
\begin{aligned}
h_{0}(x) & =\langle\delta(H-x)\rangle_{0} \\
& =\int \delta(H-x) \exp \left(-H / k T_{0}\right) \mathrm{d} \mathbf{r} \mathrm{d} \mathbf{v} / \Delta_{0} \\
h_{1}(x) & =<\delta(H-x)>_{1} \\
& =\int \delta(H-x) \exp \left(-H / k T_{1}\right) \mathrm{d} \mathbf{r} \mathrm{d} \mathbf{v} / \Delta_{1}
\end{aligned}
$$

where \langle\rangle$_{0}$ refers to an ensemble average reference state having temperature $T_{0},\langle\rangle_{1}$ to an average at $T_{1}$, and where $\delta$ is the Kroenecker delta function. The functions $h_{i}(x)$ are easily estimated in a MD sampling by compiling a histogram $h_{i}^{*}(x)$ of the frequency of occurrence of states with enthalpy $H$ between $x-\mathrm{d} x / 2$ and $x+\mathrm{d} x / 2$. Rewriting $h_{1}(x)$ as

$$
\begin{aligned}
& h_{1}(x)=\int \delta(H-x) \exp \left\{-H /\left(1 / k T_{1}-1 / k T_{0}\right)\right\} \\
& \times \exp \left[-H / k T_{0}\right] \mathrm{d} \mathbf{r} d \mathbf{v} / \Delta_{1}
\end{aligned}
$$

yields the final expression

$$
\begin{aligned}
\exp \{ & \left.-\left(G\left(T_{0}\right) / k T_{0}-G\left(T_{1}\right) / k T_{1}\right)\right\}=\Delta_{0} / \Delta_{1} \\
& =\exp \left\{-x\left[\left(1 / k T_{0}\right)-\left(1 / k T_{1}\right)\right]\left\{h_{1}(x) / h_{0}(x)\right\}\right.
\end{aligned}
$$

Note that this method requires at least a partial overlap of the two histograms $h_{0}^{*}$ and $h_{1}^{*}$. The above expression is then limited to temperature intervals $T_{1}-T_{0}$ for which $h_{1}^{*}$ and $h_{0}^{*}$ are defined and non-zero for some $x$. On the other hand the width of the distributions is, to the first approximation, proportional to the square root of the number of particles. Thus the amount of overlap for a given temperature interval will decrease as $N$ increases. Smaller intervals are then needed for a satisfactory accuracy. It is well known that all properties depend on the size of the system. An effective for avoiding problems associated with the narrowing of the width of the distributions for large systems, is to extrapolate the values of a property calculated on a series of small systems to the infinite system limit. This will provide the most accurate evaluation of the bulk property. 


\section{RESULTS}

In this section we present the results of our simulations of 2- and 3-dimensional $\Sigma=7$ bicrystals.

\subsection{The two-dimensional bicrystal}

We investigated a two-dimensional $\Sigma=7(\theta=$ $38.21^{\circ}$ ) tilt boundary in the [111] plane with 112 particles interacting through a Lennard-Jones potential

$$
\Phi(r)=4 \epsilon\left\{(\sigma / r)^{12}-(\sigma / r)^{6}\right\}
$$

with parameters chosen to simulate argon ( $\epsilon=$ $119.79 \mathrm{~K}$ and $\sigma=3.405 \mathrm{~A}$ ). The interatomic potential was truncated between second and third nearest neighbours (range of interaction $R_{i}=2.1 \sigma$ ). Because of the periodic border conditions the simulated system is really a stack of infinitely long thin crystals and the simulation cell contains a second grain boundary at the upper and lower borders in addition to the middle boundary. The bicrystal was under a reduced isostatic pressure $P^{*}=P \sigma^{2} / 4 k \epsilon=0.4936$ and each simulation lasted 10,000 time steps (a step being $0.002 \zeta$, with the characteristic time $\zeta=2.156 \times$ $\left.10^{12} \mathrm{~s}\right)$. Enthalpy and volume were calculated in a wide range of temperatures up to the bulk melting temperature $T_{\mathrm{m}}^{*}=T / 4 \epsilon=0.145$. At the lowest temperature, $T^{*}=0.055$, the determinant technique was used to obtain the absolute free energy of the bicrystal which served as the starting point for the computer calorimetry. An illustration of the relaxed low temperature structure of a $\Sigma=7$ twodimensional bicrystal having 504 atoms is shown in Fig. 1.

To calculate the excess properties of crystalline defects, we must also establish the thermodynamics of a perfect crystal reference system similar in shape and size to the defected crystal. Then the excess thermodynamic properties of the defect are found by taking the difference between those of the defected system and those of the perfect crystal reference system. A two-dimensional perfect crystal in the [111] plane with 104 particles was studied previously under identical conditions [25]. The temperature dependence of the enthalpy per particle and the volume per particle for this reference crystal is linear and fitted with the least square method

$$
\begin{array}{ll}
T^{*} \in[0.055-0.075] & H^{*} / N=2.3413 T-0.2934 \\
& V^{*} / N=0.7309 T+1.0365 \\
T^{*} \in\left[0.080-T_{\mathrm{m}}^{*}\right] & H^{*} / N=2.7106 T-0.3228 \\
& V^{*} / N=0.9752 T+1.0166 .
\end{array}
$$

In the last fitting, we discarded the points $T^{*}>0.13$ because at those temperatures spontaneous generation of defects was observed; a phenomenon not observed in the bicrystal. The "knee" we introduce in the temperature dependence of the volume and enthalpy per particle of the perfect crystal by the fitting to two linear portions does not cause a singularity in

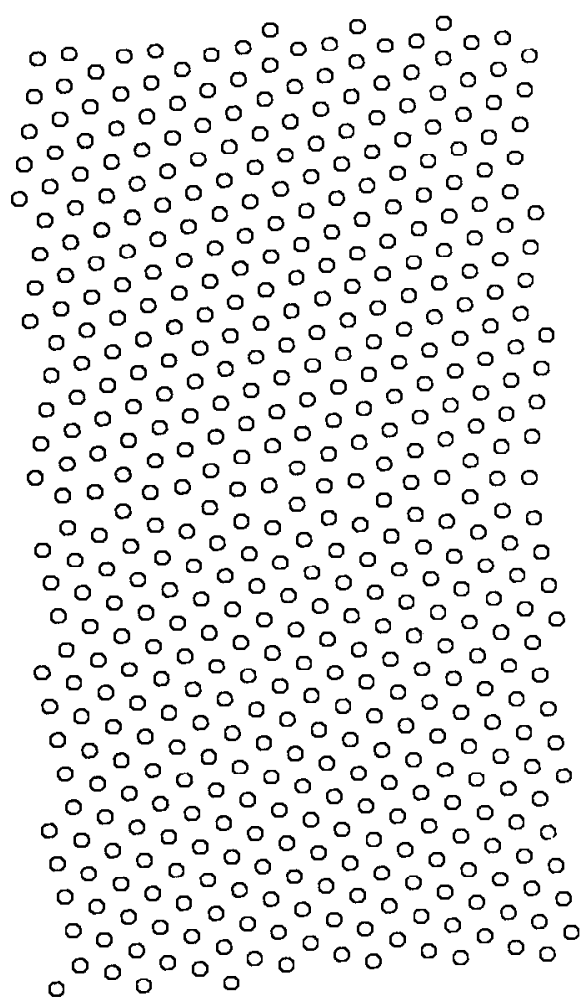

Fig. 1. Low temperature relaxed two-dimensional $\Sigma=7$ bicrystal having 504 particles.

the temperature dependence of the excess volume and excess enthalpy of the grain boundary because we do not have any data point for the thermodynamic properties of the bicrystal in the interval of temperature $0.075<T^{*}<0.085$.

The calorimetric Gibbs free energy is taken as the reference free energy for temperatures below $T^{*}=$ 0.13 . The temperature dependence of the Gibbs free energy can be calculated by traditional thermodynamic expressions if it is written as an integral over the temperature of the differential quantity $\mathrm{d} G=$ $\mathrm{d} H-\mathrm{d}(T S)$ along a reversible path linking the two extreme states. Performing the integration leads to

$$
G(T)-G\left(T_{0}\right)=H(T)-H\left(T_{0}\right)-T \int_{T_{0}}^{T} \mathrm{~d} S .
$$

The heat capacity is defined as $C_{\mathrm{p}}=T(\partial S / \partial T)_{\mathrm{p}}$. Thus the above expression becomes

$$
G(T)=H(T)-T\left\{S\left(T_{0}\right)+\int_{T_{0}}^{T}\left(C_{\mathrm{p}} / \theta\right) \mathrm{d} \theta\right\} .
$$

When the temperature interval is small, the heat capacity can be approximated by $\Delta H / \Delta T=C_{\mathrm{p}}$. We have applied this approach to the calculation of the Gibbs free energy above the temperature $T^{*}=0.13$. We have used these data as reference for the calculation of the excess Gibbs free energy of the bicrystal. the excess thermodynamic proplerties per unit length of grain boundary are collected in Table 1. Excess enthalpy, volume and Gibbs free energy are plotted respectively in Figs 2, 3 and 4. 


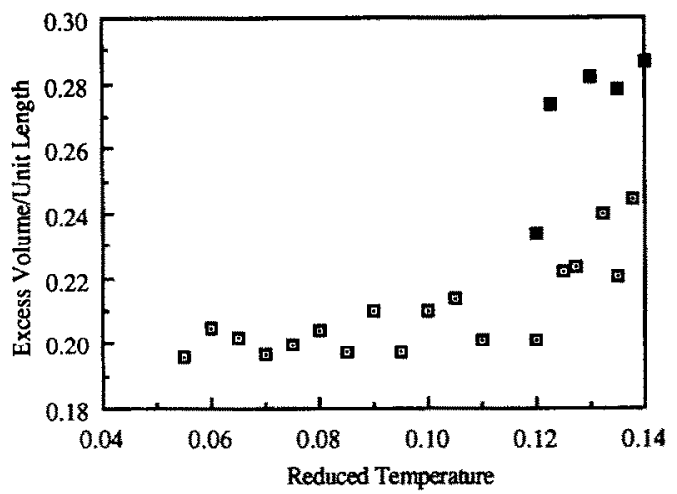

Fig. 2. Excess volume/unit length of boundary vs temperature in the case of a $2 D$ bicrystal The " $\square$ " are data corresponding to a crystalline interface, the " $\square$ " represent data for a liquid-like boundary.

Two sets of data are listed. In the simulations marked with a ".", the $T^{*}=0.055$ average structure was used as the initial configuration. The expansion

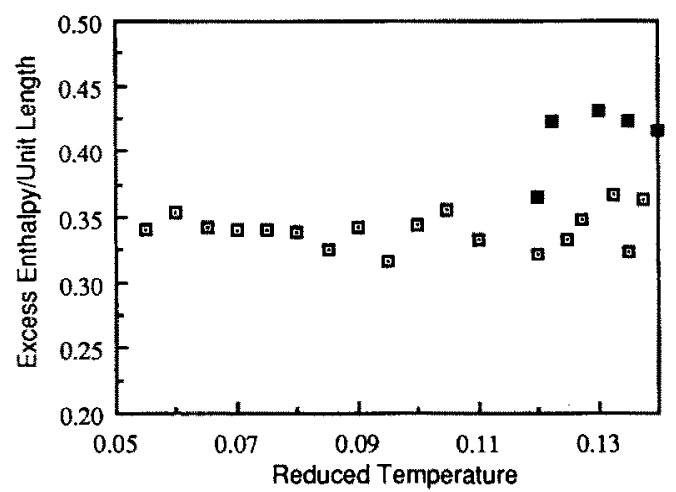

Fig. 3. Excess enthalpy per unit length vs. temperature. The " $\square$ " and the "D" correspond to a crystalline interface and a liquid-like layer respectively.

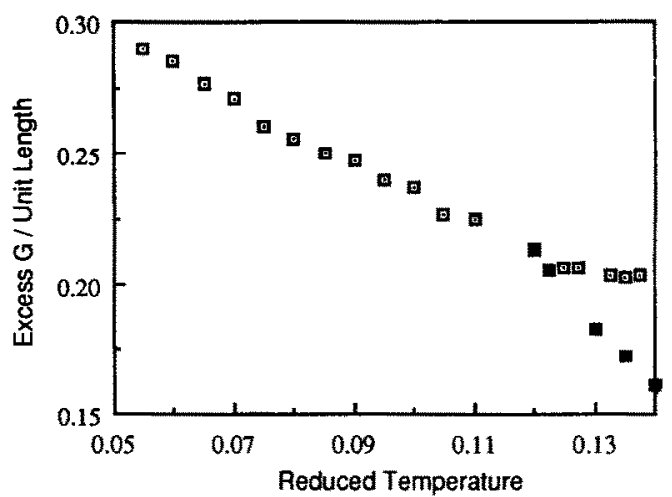

Fig. 4. Excess Gibbs free energy per unit length vs temperature. The last five data marked " $\square$ ", which were obtained from metastable crystalline boundaries, are uncertain.

to the equilibrium volume at higher temperatures took place within the first 200 time steps, or within approximately one vibrational period. The data marked with a "*" were obtained from simulations which took as a starting structure the $T^{*}=0.055$ average structure which had been given a presimulation expansion to an intermediate volume. In effect, this is a more gentle way of heating the system. When we examine the first set of data, we see a discontinuity in excess enthalpy and volume at $T^{*}=0.120$. At this temperature the grain boundary transforms fromn a crystalline to a highly disordered structure. An instantaneous snapshot of the high temperature structure of a $\Sigma=7$ bicrystal having 504 particles is shown in Fig. 5. The calculated excess Gibbs free energy of the boundary remains continuous through this transition, as shown in Fig. 4. When we employed the second starting procedure, we were able to keep the boundary in a metastable

Table 1. Excess thermodynamic properties per unit length of grain boundary in the case of a $2 D$ Lennard Jones $\Sigma=7$ bicrystal

\begin{tabular}{|c|c|c|c|c|c|c|c|c|}
\hline & $T^{*}$ & $V_{c} / L^{*}$ & $H_{\mathrm{e}} / L^{*}$ & $S_{\mathrm{e}} / L^{*}$ & $F_{\mathrm{e}} / L^{*}$ & $G_{\mathrm{e}} / L^{*}$ & & \\
\hline 1 & 0.055 & 0.1956 & 0.3402 & 0.9127 & 0.1934 & 0.2900 & & - \\
\hline 2 & 0.060 & 0.2045 & 0.3539 & 1.145 & 0.1842 & 0.2852 & & • \\
\hline 3 & 0.065 & 0.2016 & 0,3430 & 1.0169 & 0.1774 & 0.2769 & & - \\
\hline 4 & 0.070 & 0.1967 & 0.3408 & 0.9943 & 0.1741 & 0.2712 & & • \\
\hline 5 & 0.075 & 0.1995 & 0.3401 & 1.0653 & 0.1617 & 0.2602 & & - \\
\hline 6 & 0.080 & 0.2037 & 0.3387 & 1.0362 & 0.1552 & 0.2558 & & . \\
\hline 7 & 0.085 & 0.1972 & 0.3248 & 0.8718 & 0.1534 & 0.2507 & & . \\
\hline 8 & 0.090 & 0.2101 & 0.3423 & 1.0489 & 0.1442 & 0.2479 & + & • \\
\hline 9 & 0.095 & 0.1971 & 0.3154 & 0.7937 & 0.1427 & 0.2400 & & • \\
\hline 10 & 0.100 & 0.2097 & 0.3437 & 1.067 & 0.1335 & 0.2370 & + & - \\
\hline 11 & 0.105 & 0.2141 & 0.3553 & 1.2228 & 0.1212 & 0.2269 & & • \\
\hline 12 & 0.110 & 0.2007 & 0.3326 & 0.9818 & 0.1255 & 0.2246 & + & $\bullet$ \\
\hline 13 & 0.120 & 0.2008 & 0.3226 & 0.9083 & 0.1145 & 0.2136 & + & $*$ \\
\hline 14 & 0.125 & 0.2221 & 0.3339 & 1.0200 & 0.0968 & 0.2064 & & $*$ \\
\hline 15 & 0.1275 & 0.2236 & 0.3484 & 1.1137 & 0.0960 & 0.2064 & + & * \\
\hline 16 & 0.1325 & 0.2399 & 0.3664 & 1.2309 & 0.0849 & 0.2033 & + & * \\
\hline 17 & 0.135 & 0.2206 & 0.3244 & 0.9030 & 0.0936 & 0.2025 & & * \\
\hline 18 & 0.1375 & 0.2447 & 0.3631 & 1.1578 & 0.0831 & 0.2039 & + & $*$ \\
\hline \multicolumn{9}{|l|}{19} \\
\hline 20 & 0.120 & 0.2338 & 0.3647 & 1.2692 & 0.0970 & 0.2124 & + & - \\
\hline 21 & 0.1225 & 0.2734 & 0.4225 & 1.7763 & 0.0700 & 0.2049 & + & - \\
\hline 22 & 0.130 & 0.2820 & 0.4315 & 1.9146 & 0.0434 & 0.1826 & & • \\
\hline 23 & 0.135 & 0.2781 & 0.4231 & 1.8578 & 0.0350 & 0.1723 & & * \\
\hline 24 & 0.140 & 0.2864 & 0.4162 & 1.8228 & 0.0196 & 0.1610 & & - \\
\hline
\end{tabular}

The excess Gibbs free energies markied with a " + " were calculated with reference Gibbs free energies obtained by linear interpolation of the points computed by computer calorimetry. (See text for an explanation of the data marked with a "*" or a "*".) 


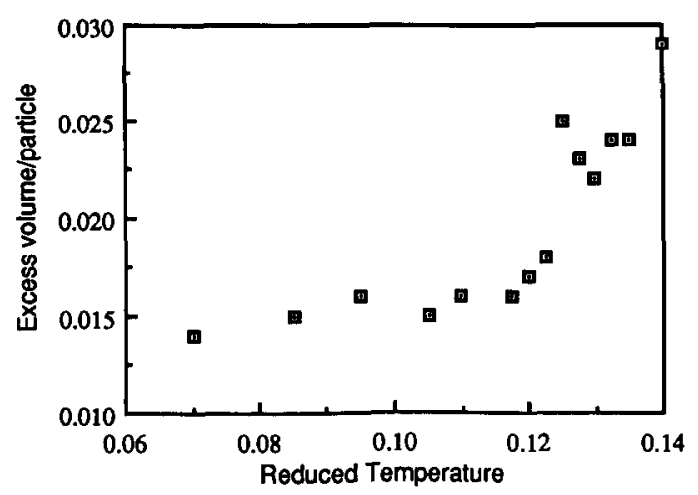

Fig. 5. Excess volume/particle vs temperature for the large (504 particle) system.

crystalline state for a few thousands steps which was a long enough period to compute averages with a satisfactory accuracy. However, it was not a long enough time for the system to sample a number of states large enough to place much confidence in the calculated free energy of the metastable crystalline boundary. A thermodynamic self-consistency check can be made by computing the excess enthalpy from the excess Gibbs free energy vs. temperature plot by using the expression $G^{\mathrm{e}}+T \partial G^{\mathrm{e}} / \partial T$. The selfconsistency check works well at temperatures below the grain boundary transition temperature. However, above the transition temperature the slope of the excess Gibbs free energy versus temperature curve gives an excess entropy approximately equal to zero for the metastable crystalline grain boundary, which is surely not a realistic situation. As was explained earlier, we cannot place too much confidence in the excess free energy of the metastable crystalline grain boundary. The self-consistency check, when applied to the liquid-like boundary gives an overestimation of the value of the enthalpy. The overestimated enthalpy is, however, well within the statistical error. In all but one of the cases in which a metastable crystaline boundary was retained the two crystalline boundaries present in the system eventually migrated toward each other and mutually annihilated, leaving behind a perfect crystal. In one case, however, we were able to retain the two metastable crystalline boundaries at $T^{*}=0.1375$ for approximately 5000 timesteps at which point the boundaries melted. This temperature was the highest one at which we were able to retain a metastable crystalline boundary.

The thermodynamic data we have plotted in Figs 2,3 , and 4 are excess quantities per unit length of boundary. The excess enthalpy per unit length of the crystalline grain boundary is essentially constant over the range of temperature studied, thus we can write $\partial\left(H^{\mathrm{e}} / L\right) / \partial T=0$. The heat capacity of the grain boundary is extracted from this equation. It is given by $C_{\mathrm{p}}=\left(\partial H^{\mathrm{e}} / \partial T\right)_{\mathrm{p}}=\left(H^{\mathrm{e}} / L\right) \cdot(\partial L / \partial T)$. The temperature dependence of the length of one grain boundary period is linear with $\Delta L / \Delta T=1.1575$ and therefore the heat capacity of one period is constant and equal to 0.39 over the temperature interval studied. The grain boundary heat capacity is indeed the difference between the heat capacity of the perfect crystal and of the bicrystal. The small heat capacity shows that the vibrational frequency spectrum of the bicrystal differs only slightly from the frequency spectrum of the perfect crystal and that only a few vibrational modes are altered by the $\Sigma=7$ grain boundary. This result is consistent with what has been observed experimentally in metals [26].

In order to check the number dependence of our results, we performed a series of similar simulations on a larger 2-dimensional $\Sigma=7$ bicrystal. The larger system contained 504 particles, and was three times as long in the direction of the boundary as the initial system. The simulations were also performed with a longer interaction range, $r_{\mathrm{i}}=2.7 \sigma$. The results plotted in Figs 5 and 6 are the excess volume per particle and the excess enthalpy per particle respectively. It should be noted that the change in interaction range slightly alters the numerical values of the calculated properties. The qualitative behaviour of the larger system is, however, identical to that of the smaller one. We observe again a discontinuity in the calculated excess properties occuring in the same temperature region. Structural changes occurring at the boundary concomitant to the phase transition are also unaltered. The results are therefore consistent with those from the small system.

We believe that the $\Sigma=7$ grain boundary underwent a first order phase transition at a temperature lower than the bulk melting temperature. At the transition temperature the crystalline grain boundary is replaced by a highly disordered liquid-like laycr. The boundary transition temperature $T_{\mathrm{m}}^{*}(\mathrm{~GB})=\mathbf{0 . 1 2}$ is about $83 \%$ of the melting point. Two major arguments support the first order character of the boundary transition in a $2 \mathrm{D}$ bicrystal. Firstly, the excess enthalpy and volume vs temperature are discontinuous at the boundary transition point. Secondly, a metastable crystalline boundary could be retained at temperatures above the calculated grain boundary transition point which would not be possible if the transformation was of higher order.

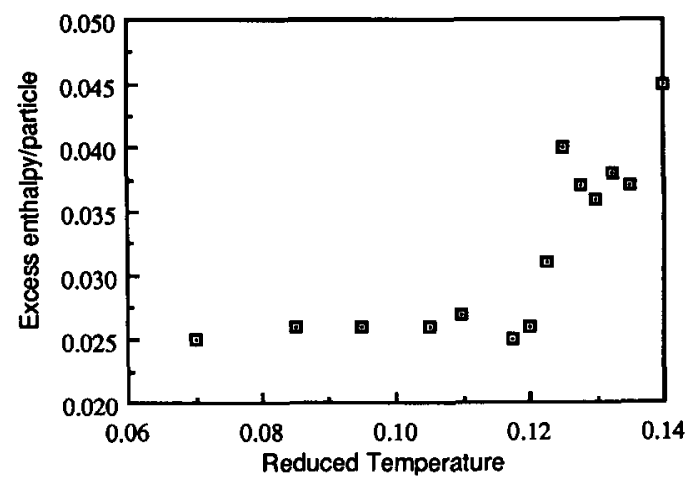

Fig. 6. Excess enthalpy/particle vs temperature for the large system. 


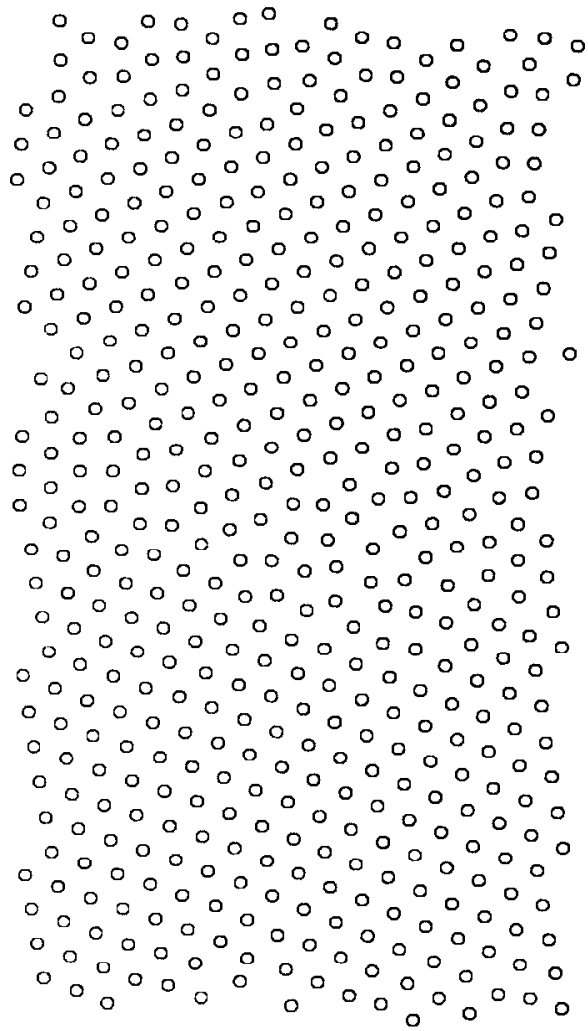

Fig. 7. High temperature $\left(T^{*}=0.1375\right) \quad \Sigma=7$ bicrystal. Each grain boundary has been replaced by a very mobile liquid-like layer.

\subsection{The three-dimensional bicrystal}

To bring our simulations closer to the real world of physical grain boundaries we moved on to study a three-dimensional $\Sigma=7\left(\theta=38.21^{\circ}\right)$ tilt boundary with tilt axis parallel to the $\langle 111\rangle$ direction. 648 particles were interacting through a Lennard-Jones potential truncated between third and fourth nearest neighbours (range of interaction: $R_{\mathrm{i}}=2.15$ ). A reduced isostatic pressure $P^{*}=P \sigma^{3} / 4 k \epsilon=0.1$ was applied and each simulation lasted 2500 steps.

We obtained the low temperature relaxed structure of the system by holding it at $T^{*}=0.01$ and calculating the time averaged atomic coordinates. We started all the simulations at $T^{*}=0.01$ with this relaxed low temperature structure as initial configuration. This temperature was maintained for 100 time steps. the system was then brought gently to the

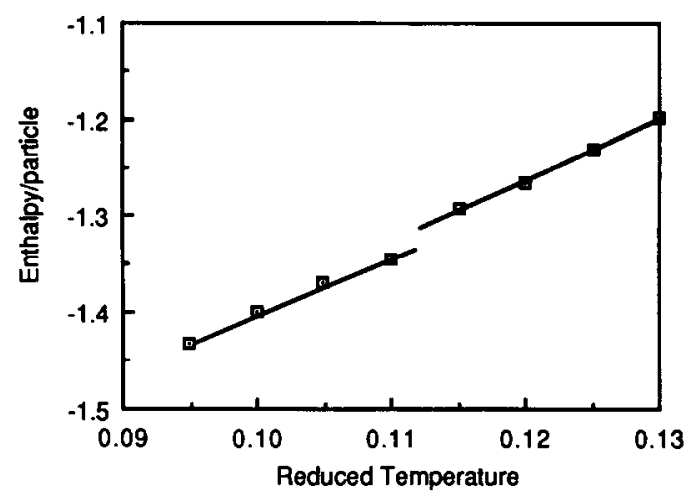

Fig. 8. Temperature dependence of the enthalpy per particle of the $3 D$ bicrystal.

desired temperature during the next $\mathbf{4 0 0}$ time steps. In Table 2, we present the thermodynamic data of the bicrystal. the determinant technique was not applied because of limitation in computational capacity; it would have required the calculation of the determinant of a $1941 \times 1941$ force constant coefficient matrix. Therefore, to overcome the lack of free energy values, we assumed

$$
G^{*}\left(T^{*}=0.095\right)=H^{*}(0.095)
$$

as initiating point for the computer calorimetry.

The bicrystal exhibits a discontinuity in its enthalpy and volume at the temperature $T^{*}=0.1125$, as shown in Figs 7 and 8 . This temperature is approximately $76 \%$ of the bulk melting point. At this temperature, instantaneous snapshots of the bicrystal indicate a disordering of the atoms in the interfacial region. The Gibbs free energy vs temperature curve, plotted in Fig. 9, exhibits only a change in slope when the boundary transforms from a crystalline state to a liquid-like layer. The Gibbs free energy of the bicrystal with an ordered or liquid-like boundary is extended through the whole temperature region by integrating the differential equation: $\mathrm{d} G=\mathrm{d} H-\mathrm{d}(T S)$ along a thermodynamically reversible path. Table 3 lists the results of this procedure. We use a linear fitting for the enthalpy temperature dependence of the ordered phase

$$
H^{*}\left(T^{*}\right) / N=5.850 T^{*}-1.9877
$$

and of the bicrystal with melted boundary

$$
H^{*}\left(T^{*}\right) / N=6.450 T^{*}-2.0352 \text {. }
$$

Table 2. Thermodynamic data of the $3 D \Sigma=7$ bicrystal

\begin{tabular}{cccccccc}
\hline & $T^{*}$ & $V^{*}(N)$ & $H^{*}(N)$ & $S^{\prime *}(N)$ & $F^{* *}(N)$ & $G^{\prime *}(N)$ \\
\hline 1 & 0.095 & 1.0429 & -1.4320 & 0.0000 & -1.5363 & -1.4320 & 33.09 \\
2 & 0.100 & 1.0540 & -1.3998 & 0.330 & -1.5382 & -1.4328 \\
3 & 0.105 & 1.0630 & -1.3691 & 0.6295 & -1.5415 & -1.4352 \\
4 & 0.110 & 1.0703 & -1.3446 & 0.8582 & -1.5460 & -1.4390 & 33.31 \\
5 & 0.115 & 1.0899 & -1.2929 & 1.3177 & -1.5334 & -1.4444 & 34.75 \\
6 & 0.120 & 1.1017 & -1.2639 & 1.5650 & -1.5619 & -1.4517 & 34.21 \\
7 & 0.125 & 1.1148 & -1.2296 & 1.8448 & -1.5717 & -1.4602 \\
8 & 0.130 & 1.1270 & -1.1967 & 2.1031 & -1.5828 & -1.4701 \\
\hline
\end{tabular}

The primed data are not absolute values but are obtained assuming $G^{*}\left(T^{*}=0.095\right)=H^{*}\left(T^{*}=0.095\right)$. A* stands for the area of the grain boundary. 


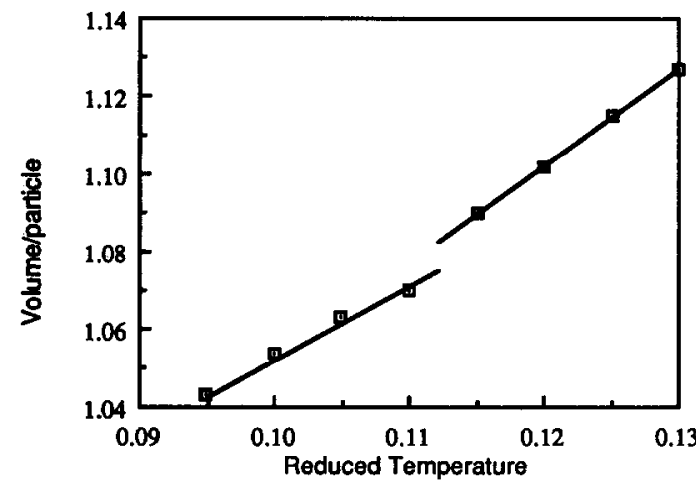

Fig. 9. Temperature dependence of the volume per particle of the $3 D$ bicrystal.

Table 3. Gibbs free energies of the $3 D$ bicrystal with crystalline grain boundaries $\left[\mathrm{G}(\mathrm{c})^{*}\right]$ and of the $3 D$ bicrystal with liquid-like layers $\left[G(1)^{*}\right]$ extended to the whole range of temperatures by integrating the differential equation: $\mathrm{d} G=\mathrm{d} H-\mathrm{d}(T S)$

\begin{tabular}{cccc}
\hline & $T^{*}$ & $G(c)^{*}(N)$ & $G(I)^{*}(N)$ \\
\hline 1 & 0.095 & -1.4320 & -1.4300 \\
2 & 0.100 & -1.4328 & -1.4313 \\
3 & 0.105 & -1.4350 & -1.4341 \\
4 & 0.110 & -1.4386 & -1.4385 \\
5 & 0.115 & -1.4435 & -1.4444 \\
6 & 0.120 & -1.4497 & -1.4516 \\
7 & 0.125 & -1.4572 & -1.4602 \\
8 & 0.130 & -1.4658 & -1.4701 \\
\hline
\end{tabular}

The theoretical curves agree extremely well with the computed points and intersect where the boundary undergoes the transition. At low temperature, the liquid like layer is unstable with respect to the crystalline boundary. At the crossing point the tendency is inverted and the liquid like boundary becomes stable. The thermodynamic behavior of a grain boundary in a 3-dimensional bicrystal at high temperature demonstrates the first order character of the grain boundary transition.

\section{DISCUSSION AND CONCLUSION}

In this paper we have reported the results of a thorough study, by molecular dynamics, of the thermodynamic behaviour of 2- and 3-dimensional $\Sigma=7$ grain boundaries. For both boundaries, we observed a grain boundary phase transition in which a well-ordered crystalline boundary was replaced by a highly disordered liquid-like layer. The transition takes place at 83 and $76 \%$ of the bulk melting points in 2- and 3-dimensional bicrystals respectively. The transition is first order, in both cases. This is clear from the sharp discontinuities in the calculated enthalpies and volumes. Another indication of the first order nature of the transition is the fact that we were able to retain a metastable crystalline boundary above the boundary melting temperature, in the $2 D$ case.

At this point, it would be useful to compare simulation results with some recent experimental work. A study of an off-coincidence $\Sigma=5$ tilt grain

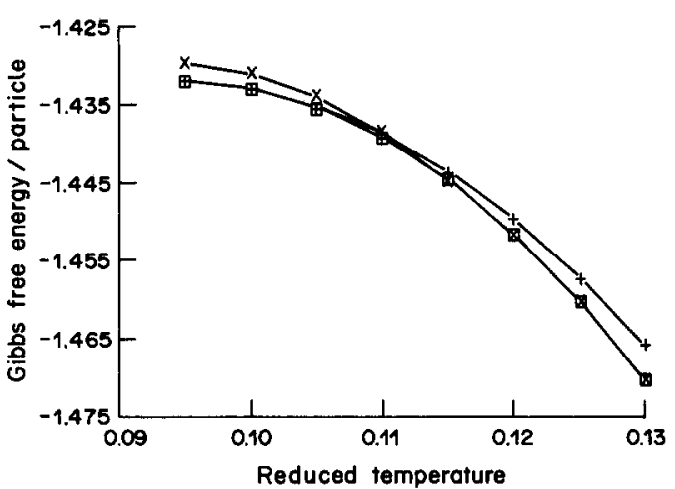

Fig. 10. Temperature dependence of the Gibbs free energy per particle of the $3 D$ bicrystal. The " $\square$ " are data points obtained by computer calorimetry. Those marked " + " correspond to a thermodynamic extension of the crystalline free energy in the high temperature region. Those marked " $x$ " conversely represent a luw temperature extension of the free energy of the bicrystal with melted boundaries.

boundary in aluminum was performed using transmission electron microscopy at elevated temperatures [27]. The secondary dislocation network of the boundary was still visible at $92 \%$ of the bulk melting temperature, from which it seems clear that the boundary had not melted. Very recently a similar aluminum grain boundary was studied using molecular dynamics [16]. A $\Sigma=5$ symmetrical tilt grain boundary with tilt axis along the [001] direction was replaced by a highly disordered melted layer at about $70 \%$ of the bulk melting temperature. Is the grain boundary phase transition a natural phenomenon or only a property of models of grain boundaries in computer simulations? Differences between experiments and molecular dynamics simulation that might contribute to the contradiction between experiments and simulations should be pointed out at this stage. The interatomic potentials used in the computer simulations do not seem to have a major effect on the behaviour of the grain boundary. The $\Sigma=5$ in $\mathrm{Al}$ exhibited a melting transition in simulations conducted with a Morse potential and a pseudopotential. We have observed phase transitions in all the boundaries we have studied using a Lennard-Jones potential. There are also the effects of the very limited system sizes accessible in the simulations. These effects, however, do not seem to have any major consequences for the melting transition. Another major difference between experiment and simulations is that point defects are always present in the physical bicrystals but not always in the simulated bicrystals. A grain boundary is an open system and is free to exchange point defects with the grains to minimize its free energy. Point defects will add a great deal of configurational entropy to the grain boundary and may be capable of stabilizing it with respect to other grain boundary phases. One might expect a grain boundary melting transition in this grain boundary with point defects to take place at a higher temperature than that of the perfect grain boundary. 
The calculated thermodynamic properties of our systems demonstrate conclusively the presence of a phase transition at our grain boundaries. The question of whether what we call a grain boundary melting transition in this study is a true melting transition or some other kind of grain boundary phase transition is still open. If the transition is a true melting transition, the calculated excess free energy should vanish as the bulk melting point is approached. We are unable to characterize the properties of the transformed layer at temperatures greater than approximately $90 \%$ of the bulk melting point. At higher temperatures the system is able to rid itself of its boundaries at time scales which are too small for meaningful thermodynamic averages to be taken. The nature of the phase transition could be further elucidated by additional work on the structural characterization of the transformed layer. Though such characterization is made cumbersome by the high mobility of the layer at elevated temperatures, it is certainly not impossible, and further work will be done. In simulation studies of the $\Sigma=5$ symmetrical tilt boundary using a Morse potential for aluminium more detailed structural characterization has been performed [16]. The structure factor and mean square displacements were calculated in a series of layers parallel to the boundary, and were observed to approach the values characteristic of bulk undercooled liquids after the grain boundary phase transition occurred. We have performed diffraction studies on another tilt boundary, the $\Sigma=13$, with the Lennard-Jones potential [28]. The diffraction patterns of the bicrystal after the transition has occurred are a superposition of the diffraction pattern of two misoriented grains and that of a liquid. It now seems reasonable to assume that the first order transition we observe at our grain boundaries is indeed a grain boundary melting transition.

We implemented a number of techniques for the calculation of free energies of our systems using molecular dynamics trajectories. Using these techniques we were able to calculate Gibbs free energy throughout the temperature range for both the $2 D$ and the $3 D$ boundaries. The calculated Gibbs free energy vs temperature curves for the crystaline and liquid-like grain boundary phases intersect at the observed grain boundary melting point.

Each of the techniques we employed for calculation of grain boundary excess free energy has intrinsic limitations and advantages. The determinant technique is only useful when employed at temperatures low enough to avoid anharmonic contributions to the free energy, but high enough to avoid quantum effects. There are also other restrictions on its use. The technique is based on the generation of an average structure, from which the force constant coefficient matrix may be computed. When molecular dynamics simulations are conducted at high temperature, and when crystalline defects such as grain boundaries are present, the high mobility of the defects may render any average structure physically meaningless, and useless for the determinant technique. Related to this difficulty is the fact that the determinant technique only accesses the vibrational contribution to the entropy; no contribution to configurational entropy can be examined. Some of the restrictions inherent to the determinant technique can be lifted by going to the energy distribution method we have described. Computer calorimetry allowed us to compute the free energy of our system throughout the temperature range. The computer calorimetry technique has, however, the major limitation that it only provides estimates of free energy differences, and not absolute values of the free energies of the systems of interest. We were unable to use the determinant technique on the $3 D$ bicrystal because the size of the force constant coefficient matrix was too large for the computer to handle. Therefore we could not use the quasi-harmonic free energy to initiate the computer calorimetry, and we do not have absolute values of the 3-dimensional $\Sigma=7$ excess free energy.

To date we have very little quantitative information as to the effect our grain boundary phase transition will have on the mechanical properties of bicrystals at elevated temperatures. That topic will be the subject of future research. In the work of Watanabe et al. [11], in which they measured sliding velocity of well-prepared bicrystals as a function of temperature, they observed a sharp discontinuity in grain boundary properties. They have no high resolution in situ structural characterization of the changes occuring at the boundary during the transition. However, it is reasonable to assume that some sort of a transition is occurring, and this transition has several qualitative features similar to the transition observed in our simulations. The relative temperatures at which the discontinuity occurred agree qualitatively with our studies, as does the change in transition temperature with boundary type. In the isostressisothermal formulation of molecular dynamics we can apply shear stresses to the bicrystal parallel to the boundary plane, measure the sliding velocity of one grain with respect to the other, and determine the effect of the melting transition on the sliding velocity. We have conducted a preliminary study of mimicking their experimental study by molecular dynamics [29].

This work demonstrates the range of thermodynamic information that can be obtained by employing molecular dynamics techniques in the study of crystalline interfaces. It is part of a wider cffort to systematically characterize phase diagrams of grain boundaries. We chose a very simple interaction potential and a very simple, short period coincidence type boundary. The boundary was chosen to be at a symmetry dictated extremum with respect to facetting of the boundary plane in order to maximize the probability of observing a congruent grain boundary melting transition. Molecular dynamics will be a powerful tool to study other classes of grain boundary phase transitions, and hence to gain a more 
thorough and complete knowledge of interfacial phase equilibria.

Acknowledgements - This research was sponsored by NSF/ MRL grant No. DMR 81-19295 and through a Presidential Young Investigator Award. We would like to express our gratitude to Dr Manfred Rühle of the Institut fur Werkstoffwissenschaften, Max-Planck Institut für Metallforschung, Stuttgart, for the hospitality and computational resources extended to us during the time spent by both of us at his laboratory. We are also very grateful to Siegried Schmauder, of the same institute, for his help in computational aspects of our work. We thankfully acknowledge the contributions of Dr Reza Najafabadi, of M.I.T., in the form of programming assistance and many helpful discussions. We want also to thank J. W. Cahn of the National Bureau of Standards for his critical reading of the manuscript and for his useful comments.

\section{REFERENCES}

1. J. W. Gibbs, Collected Works, Vol. I, Longmans Green, New York (1928).

2. D. W. Hoffman and J. W. Cahn, Acta metall. 22, 1205 (1974).

3. C. Rottman and M. Wortis, Phys. Rev. 29, 328 (1984).

4. J. W. Cahn, J. Physique C6 43, 199 (1982).

5. G. Kalonji, Ph.D. thesis, M.I.T. (1982).

6. J. C. Shelton, H. R. Patil and J. M. Blakely, Surf. Sci. 43, 493 (1974).

7. M. Guttmann, Metall. Trans. 8A, 1383 (1977).

8. R. H. Willens, A. Kornblit, L. R. Testardi and S. Nakahara, Phys Rev. B25, 290 (1982).

9. M. E. Glicksman and C. L. Vold, Acta metall. 13, 1 (1969).

10. M. Biscondi and C. Goux, Mem. Scient. Revue Metall. 65, 167 (1968).
11. T. Watanabe, S. I. Kimura and S. Karashuma, Phil. Mag. A. 49, 167 (1984).

12. R. Kikuchi and J. W. Cahn, Phys. Rev. B21, 1893 (1980).

13. G. Giccotti, M. Guillope and V. Pontikis, Phys. Rev B27, 5576 (1983).

14. F. Carrion, G. Kalonji and S. Yip, Scripta metall. 17, 915 (1983).

15. G. Kalonji, P. Deymier, R. Najafabadi and S. Yip, Surf. Sci. 144, 77 (1984).

16. T. Nguyen, P. S. Ho, T. Kwok, C. Nitta and S. Yip, Phys. Rev. Lett. 57, 1919 (1986).

17. J. Q. Broughton and G. H. Gilmer, Phys. Rev. Lett. 56, 2692 (1986).

18. M. Parrinello and A. Rahman, J. appl. Phys. 52, 7182 (1981).

19. H. C. Andersen, J. chem. Phys. 72, 2384 (1980).

20. W. G. Hoover, A. C. Hindmarsh and B. L. Holian, J. chem. Phys. 57, 1980 (1972).

21. G. Venkataraman, L. A. Feldkamp and V. C. Sahni, Dynamics of Perfect Crystals, M.I.T. Press, Cambridge, Mass. (1975).

22. J. G. Kirkwood, Phys. Rev. 44, 31 (1933); 45, 116 (1934).

23. J. P. Valleau and G. M. Torrie, in Modern Theoretical Chemistry (edited by B. J. Berne). Vol 5. Plenum Press, New York (1976).

24. C. H. Bennett, J. comput. Phys. 22, 245 (1976).

25. P. Deymier and G, Kalonji, J. chem. Phys. 85, 2937-42 (1986).

26. L. E. Murr, G. I. Wong and R. J. Horylev, Acta metall. 21, 595 (1973).

27. S. W. Chan and E. W. Balluffi, Scripta metall.

28. P. Deymier and G. Kalonji, Phase Transitions of Grain Boundaries, Proc. Int. Conf. Internal Surfaces, JIMS IV, Minakami-kan, Japan (1985).

29. P. Deymier and G. Kalonji, Scripta metall, 20, 13 (1986). 